\title{
COGNIÇÃO, ASPECTOS PSICOLÓGICOS E QUALIDADE DE VIDA EM IDOSOS COM COMPROMETIMENTO COGNITIVO LEVE
}

\author{
Amanda de Oliveira Ferreira Leite ${ }^{1}$ \\ Ana Luiza Ferreira ${ }^{2}$ \\ Bruna Seling ${ }^{3}$ \\ Janaíne $\mathrm{Mello}^{4}$ \\ Marla Vieira ${ }^{5}$ \\ Mirna Wetters Portuguez ${ }^{6}$
}

resumo

O Comprometimento Cognitivo Leve (CCL) é geralmente um estado intermediário entre a cognição normal e a demência em que as habili-

1 Psicóloga. Mestre em Neurociências e Biologia Celular. Doutoranda em Gerontologia Biomédica pela Pontifícia Universidade Católica do Rio Grande do Sul e psicóloga hospitalar no Hospital Escola da Universidade Federal de Pelotas (HE - UFPEL). E-mail: amanda.neuropsi@hotmail.com

2 Aluna do curso de Psicologia da Universidade da Região da Campanha, Campus Bagé (URCAMP - Bagé). E-mail: analuizadelpino@gmail.com

3 Aluna do curso de Psicologia da URCAMP - Bagé. E-mail: nanagarcezcorrea@gmail.com

4 Aluna do curso de Psicologia da URCAMP - Bagé. E-mail: janainercc3@gmail.com

5 Aluna do curso de Psicologia da URCAMP - Bagé. E-mail: marla.manquevich@gmail.com

6 Psicóloga. Doutora em Neurociências. Professora e pesquisadora da Pontifícia Universidade Católica do Rio Grande do Sul, vinculada à Faculdade de Medicina, ao Curso de Pós-Graduação do Instituto de Geriatria (IGG) e Gerontologia e ao Instituto do Cérebro (InsCer). E-mail: mirna@pucrs.com 
dades funcionais essenciais estão preservadas. Objetivos: caracterizar uma amostra de idosos com CCL residentes no sul do Brasil quanto ao desempenho em instrumentos que avaliam cognição, aspectos psicológicos e qualidade de vida. Método: 96 idosas, entre 60 e 84 anos, foram avaliadas com os seguintes instrumentos: Exame Cognitivo de Addenbrooke-Revisado, Índice de Katz, Escala de Lawton \& Brody, Escala de Depressão Geriátrica, Inventário de Ansiedade de Beck, Escala de Desenvolvimento Pessoal, WHOQOL-bref e WHOQOL-old. O diagnóstico de CCL foi feito a partir de entrevista semiestruturada, avaliação cognitiva e funcional. $\bigcirc$ desempenho cognitivo foi ajustado conforme a idade e a escolaridade dos sujeitos. Resultados: O grupo com CCL mostrou déficit nos cinco domínios cognitivos avaliados, apresentou sintomas de ansiedade, prejuízos no bem-estar psicológico e na qualidade de vida. Conclusões: A combinação dos instrumentos utilizados neste estudo não foi feita anteriormente e explicitou os danos diversos causados pelo CCL em relação a controles saudáveis. Em geral, as pesquisas da área avaliam idosos com histórico urbano e com escolaridade e renda mais altas. Esta amostra, entretanto, caracteriza-se por escolaridade e renda familiar predominantemente baixas, além de forte tradição rural, trazendo contribuições importantes se considerarmos a heterogeneidade característica da população brasileira. Considerando que a avaliação neuropsicológica é uma ferramenta essencial para mensurar prejuízo e declínio das funções cognitivas e que não há padrão-ouro para o CCL, a metodologia proposta neste trabalho pode ser um caminho.

palavras-chave

Comprometimento Cognitivo Leve. Ansiedade. Depressão. Qualidade de Vida. Idoso.

Proporcionalmente, a população idosa é a que mais cresce no Brasil (IBGE, 2013). A maior parte dela desenvolve uma trajetória evolutiva estável e benigna, não apresentando declínio cognitivo que afete a vida cotidiana (CARLOMANHO; SOARES, 2013).

O conceito de Comprometimento Cognitivo Leve (CCL) foi criado para compreender as fronteiras entre o envelhecimento saudável e o patológico 
(STOKIN et al., 2015). O CCL é geralmente um estado intermediário entre a cognição normal e a demência em que as habilidades funcionais essenciais estão preservadas (PETERSEN et al., 2014).

A presente pesquisa é singular ao investigar uma população com dificuldades socioeconômicas e características culturais específicas. Ainda são escassos os estudos sobre o desempenho de idosos com CCL no Exame Cognitivo de Addenbrooke e no WHOQOL-old, em especial no Brasil, e não foram encontradas pesquisas que avaliassem o bem-estar psicológico desses pacientes por meio da Escala de Desenvolvimento Pessoal. Desta forma, o objetivo desta pesquisa foi caracterizar uma amostra de idosos com Comprometimento Cognitivo Leve residente no Sul do Brasil quanto ao desempenho em instrumentos que avaliam cognição, aspectos psicológicos e qualidade de vida.

\section{Metodologia \\ 2.1 Sujeitos}

O estudo foi um ensaio clínico controlado, prospectivo, composto por 96 mulheres idosas, com idade entre 60 e 84 anos, participantes de um centro de convivência para idosos. Utilizou-se a técnica de amostragem por conveniência. Os critérios de inclusão na pesquisa foram: voluntários participantes do Centro do Idoso; 60 anos ou mais; 4 anos ou mais de estudo. Os critérios de exclusão foram: quadros demenciais; doenças neurológicas ou degenerativas graves; transtornos psiquiátricos severos; não ter disponibilidade para participar das etapas do estudo. Foram realizados testes de rastreio para diagnosticar os sujeitos com Comprometimento Cognitivo Leve.

\subsection{Instrumentos}

Entrevista semiestruturada $=$ Foram coletadas as seguintes informações: sexo, idade, escolaridade, estado civil, renda, problemas de saúde, prática de atividades físicas e queixas de dificuldades cognitivas.

Exame Cognitivo de Addenbrooke = Bateria de testes breve capaz de detectar sinais iniciais de prejuízo cognitivo, além de ser útil para diagnóstico diferencial de demências (GOLD, 2012). Possui 6 subtestes (orientação, atenção, memória, fluência verbal, linguagem e habilidade visuoespacial), variando de 0 a 100 pontos. Foi utilizada a versão brasileira do instrumento (CARVALHO; CARAMELLI, 2007). 
Escalas de avaliação funcional $=$ Foi utilizado o Índice de Katz (LINO et al., 2008) para investigar as atividades básicas de vida diária e a Escala de Lawton \& Brody (SANTOS; VIRTUOSO JÚNIOR, 2008) para avaliar as atividades instrumentais da vida diária.

Escala de Depressão Geriátrica (GDS-15) = Uma das medidas mais utilizadas para o rastreamento de sintomas depressivos em idosos (DENNIS; KADRI; COFFEY, 2012). O escore total é feito a partir do somatório das respostas assinaladas nos 15 itens que compõem o instrumento (ALMEIDA; ALMEIDA, 1999).

Inventário de Ansiedade de Beck $(\mathrm{BAI})=$ É uma escala autoaplicável que mede a intensidade dos sintomas de ansiedade. Possui uma lista de 21 sintomas com quatro alternativas cada um, em ordem crescente do nível de ansiedade (CUNHA et al., 2000).

Escala de Desenvolvimento Pessoal $($ EDEP $)=$ Possui 30 itens que avaliam 6 dimensões: relações positivas com outros; autonomia; propósito de vida; crescimento pessoal; autoaceitação; domínio do ambiente; e geratividade (NERI, 2001). Cada item é avaliado por uma escala contendo cinco pontos (1 - pouquíssimo; 2 - pouco; 3 - mais ou menos; 4 - muito; 5 - muitíssimo).

WHOQOL-old = É um módulo específico de aferição de qualidade de vida em idosos da Organização Mundial de Saúde. Possui 24 itens divididos em seis domínios: 1) Funcionamento do Sensório; 2) Autonomia; 3) Atividades Passadas, Presentes e Futuras; 4) Participação Social; 5) Morte e Morrer; e 6) Intimidade. De acordo com os autores, deve ser aplicado conjuntamente com o WHOQOL-bref (FLECK et al., 2000; POWER; QUINN; SCHMIDT, 2005).

\subsection{Procedimentos de coleta e considerações éticas}

Para o rastreio do CCL, foram utilizados os seguintes critérios diagnósticos: queixas de dificuldades cognitivas; funcionamento cognitivo global intacto; ausência de déficits nas atividades de básicas e instrumentais de vida diária; desempenho abaixo da média em um ou mais domínios cognitivos, devidamente ajustados conforme a idade e a escolaridade (PETERSEN et al., 2014).

O relato das queixas cognitivas foi coletado durante a entrevista semiestruturada. As atividades básicas e instrumentais de vida diárias foram acessadas a partir do Índice de Katz e da Escala de Lawton e Brody, respectivamente. A cognição foi avaliada pelo Exame Cognitivo de Addenbrooke, considerando-se 
como desempenho abaixo da média os idosos que obtiveram pontuação inferior a 60 pontos (para sujeitos entre 4 e 7 anos de estudo), inferior a 70 pontos (entre 8 e 11 anos de estudo) e inferior a 80 pontos (para idosos com 12 anos ou mais de estudo) (CARVALHO; CARAMELLI, 2012).

Os sujeitos com desempenho cognitivo abaixo da média e que preencheram os demais critérios descritos acima compuseram o grupo com CCL, totalizando 48 idosas. Para compor o grupo controle, foram selecionados 48 participantes saudáveis; os grupos foram pareados conforme os anos de escolaridade. Os instrumentos foram aplicados com o auxílio de quatro acadêmicos de Psicologia devidamente treinados e supervisionados.

A descrição das variáveis foi realizada por meio das frequências absolutas e relativas, bem como da média e do desvio padrão. Para comparação dos grupos foram utilizados os seguintes testes: Fisher; $\mathrm{x}^{2}$ (qui-quadrado); e teste $\mathrm{t}$ para as amostras independentes. A análise dos dados foi supervisionada pela Assessoria Estatística da Faculdade de Matemática da PUCRS. Foi utilizado o programa SPSS ${ }^{\circledR}$ para ambiente Windows ${ }^{\circledR}$, versão 13.0.

O projeto foi examinado e aprovado pelo Comitê de Ética em Pesquisa da PUCRS sob o n.o 987.566 e cumpriu os princípios éticos contidos na Declaração de Helsinki, além do atendimento à legislação pertinente. Após a aprovação, a pesquisa foi realizada no Centro do Idoso, um espaço de convivência localizado em Bagé, RS. Todos os participantes assinaram o Termo de Consentimento Livre e Esclarecido após receberem informações detalhadas sobre o estudo. As informações coletadas foram tratadas como confidenciais e a identidade dos participantes foi preservada. A pesquisa foi registrada no Registro Brasileiro de Ensaios Clínicos sob o número de identificação RBR-7m7vs2.

\section{Resultados}

Na Tabela 1, observamos que todos os voluntários que participaram da pesquisa eram do sexo feminino. As idosas com CCL apresentaram média de idade maior que as saudáveis. Em ambos os grupos, a maioria das idosas tinha baixa escolaridade, praticava atividades físicas e relatou ser casada ou viúva. A renda prevalente foi de 1 a 4 salários mínimos. Quando questionadas sobre o estado atual de saúde, foi frequente o relato de diversas condições clínicas. 
Tabela 1 - Características sociodemográficas em idosas saudáveis e com CCL.

\begin{tabular}{|c|c|c|}
\hline \multicolumn{2}{|c|}{ Idade - média \pm DP: $70,27 \pm 6,98$} & \multirow{2}{*}{$\frac{\text { CCL n (\%) }}{24(50,00)}$} \\
\hline \multirow{3}{*}{ Escolaridade (anos) } & $4-7$ & \\
\hline & $8-11$ & $13(27,08)$ \\
\hline & $\geq 12$ & $11(22,92)$ \\
\hline \multirow{4}{*}{ Estado civil } & Solteiro & $7(14,58)$ \\
\hline & Casado & $14(29,16)$ \\
\hline & Separado & $7(14,58)$ \\
\hline & Viúvo & $20(41,68)$ \\
\hline \multirow{3}{*}{ Renda (salário mínimo) } & $1-2$ & $38(79,17)$ \\
\hline & $3-4$ & $9(18,75)$ \\
\hline & $\geq 5$ & $1(2,08)$ \\
\hline \multirow{2}{*}{ Problemas de saúde } & Sim & $35(72,92)$ \\
\hline & Não & $13(27,08)$ \\
\hline \multirow{2}{*}{ Atividade física } & $\operatorname{Sim}$ & $45(93,75)$ \\
\hline & Não & $3(6,25)$ \\
\hline
\end{tabular}

Nota: ${ }^{*}$ Teste $t ;$ **Teste qui-quadrado; ${ }^{\star \star \star}$ Teste de Fisher.

Na Tabela 2, estão expostas as médias e desvio padrão obtidos pelos controles e pelos sujeitos com CCL no ACE-R. Tanto nas cinco subescalas quanto na pontuação geral, as idosas com CCL mostraram desempenho significativamente inferior $(p<0,0001)$.

Tabela 2 - Desempenho da amostra no Exame Cognitivo de Addenbrooke.

\begin{tabular}{l|c}
\multicolumn{1}{c|}{ Subtestes - média \pm DP } & CCL \\
\hline Atenção e orientação & $13,17 \pm 2,38$ \\
\hline Memória & $13,77 \pm 3,56$ \\
\hline Fluência verbal & $6,15 \pm 2,82$ \\
\hline Linguagem & $16,90 \pm 4,06$ \\
\hline Habilidades visuoespaciais & $11,67 \pm 2,46$ \\
\hline Pontuação total & $61,65 \pm 10,61$ \\
\hline
\end{tabular}

Nota: Teste t para amostras independentes $(p<0,0001)$. 
Na Tabela 3, verifica-se que as idosas com CCL tiveram pior desempenho em quatro das cinco escalas avaliadas: Inventário de Ansiedade de Beck, Escala de Desenvolvimento Psicológico, WHOQOL-bref e WHOQOL-old. Na Escala de Depressão Geriátrica, os dois grupos apresentaram sintomatologia leve de depressão.

Tabela 3 - Instrumentos de avaliação psicológica e de qualidade de vida.

\begin{tabular}{l|c|c|c}
\hline \multicolumn{1}{c|}{ Teste - média (DP) } & CCL & Controles & $\boldsymbol{p}^{*}$ \\
\hline Escala de Depressão Geriátrica & $3,69(3,33)$ & $3,04(2,23)$ & 0,13 \\
\hline Inventário de Ansiedade de Beck & $9,04(1,06)$ & $5,44(0,47)$ & 0,02 \\
\hline Escala de Desenvolvimento Pessoal & $121,47(11,37)$ & $125,00(8,73)$ & 0,05 \\
\hline WHOQOL-bref & $3,52(0,54)$ & $3,75(0,72)$ & 0,04 \\
\hline WHOQOL-old & $92,95(9,66)$ & $95,29(8,50)$ & 0,05 \\
\hline
\end{tabular}

Nota: ${ }^{\star}$ Teste t para amostras independentes.

Em nossa amostra, todos os voluntários foram do sexo feminino. A expressiva participação de mulheres é um fenômeno recorrente em estudos que envolvem grupos de convivência de idosos (KRUG; LOPES; MAZO, 2014). Algumas hipóteses para explicar essa diferença são: maior prevalência em idosas de condições incapacitantes não fatais (como osteoporose e depressão); maior habilidade em reportar problemas de saúde quando comparadas a homens da mesma idade; e maior consciência sobre a importância da atividade física para a qualidade de vida.

Além disso, muitos homens veem a aposentadoria como oportunidade ideal para passar mais tempo em casa, ver os amigos ou se distrair (BATISTA; VIEIRA; SILVA, 2012). Na idade avançada, inúmeros homens necessitam de tratamento médico para quadros irreversíveis de adoecimento, em virtude da ausência de medidas preventivas e de tratamento precoce das enfermidades. Dessa forma, ações destinadas aos idosos também devem considerar alternativas que atraiam o público masculino, fomentando a interação social, a informação, o lazer e a qualidade de vida (BATISTA; VIEIRA; SILVA, 2012). 
Neste estudo, as idosas com declínio cognitivo apresentaram maior média de idade. Pacientes mais velhos e com menor escolaridade tendem a apresentar menor desempenho nos subtestes cognitivos do ACE-R (CARVALHO; CARAMELLI, 2012); são necessários estudos que avaliem o desempenho de sujeitos com menos de 4 anos de educação formal (CARVALHO; CARAMELLI, 2012).

Mais de $50 \%$ da amostra tinha apenas entre 4 e 7 anos de escolaridade. É importante frisar que a amostra foi predominantemente originária da zona rural do município de Bagé, apesar de atualmente residir na zona urbana. $\mathrm{O}$ nível de escolaridade representa um dos indicadores mais importantes das condições de saúde e está altamente relacionado à idade no Brasil, posto que muitos idosos deste século não tiveram oportunidades de estudo quando jovens (BARROS et al., 2011). Sabe-se, por exemplo, que quanto menor o tempo de educação formal, maior a prevalência de hipertensão, o que evidencia a desigualdade em saúde, considerando-se que idosos com pouca escolaridade têm acesso precário ao sistema de saúde, recebem menos informações e muitas vezes não compreendem a importância do problema e da adesão ao tratamento, demonstrando a importância de ações voltadas para a prevenção (ZAVATINI; OBRELI NETO; CUMAN, 2010).

Quanto ao estado civil, observamos que a maioria das idosas era viúva, seguido pelas idosas casadas. A maior parte das idosas viúvas morava sozinha, as quais relataram sentirem-se mais alegres e bem-dispostas depois que passaram a frequentar o centro de convivência. As mulheres casadas, por sua vez, afirmaram que os maridos não demonstram interesse em participar das atividades do Centro, apesar delas ressaltarem os benefícios envolvidos. As mulheres tendem a continuar sozinhas, enquanto os homens, em geral, casam-se novamente; são comuns queixas de solidão, entretanto também há idosas que consideram a viuvez como uma possibilidade de ter mais liberdade e autonomia (SMAGULA et al., 2016).

A renda da maioria das participantes ficou na faixa de 1 a 2 salários mínimos. Os estudos sobre a influência da renda na capacidade funcional divergem, pois alguns apontam forte associação entre boa condição financeira e melhor capacidade funcional, enquanto outros destacam que $30 \%$ dos idosos com baixa renda apresentam altos níveis funcionais em comparação aos que possuem uma renda alta (REIS; REIS; TORRES, 2015). De fato, as idosas com menor poder aquisitivo necessitam executar as tarefas domésticas e familiares sozinhas, pois não podem contratar ajudantes; isso as manteria ativas e funcionais por mais tempo (REIS; REIS; TORRES, 2015).

Uma parcela significativa das participantes não se descreveu como saudável, destacando-se doenças como hipertensão e cardiopatias. Em todo o mundo, 
observa-se alta prevalência da hipertensão arterial, sendo esse o distúrbio cardiovascular de maior prevalência no Brasil: mais de 55\% da população acima de 60 anos tem a doença (MENDES; MORAES; GOMES, 2014).

Como nossa amostra caracteriza-se por morar em uma região de forte tradição rural, infere-se que eles desenvolveram trabalhos físicos desgastantes e não tiveram acesso frequente a serviços de saúde e, consequentemente, relatam mais doenças. Quanto menor o nível socioeconômico, pior a saúde: a pobreza, a baixa escolaridade e o baixo nível ocupacional expõem os idosos ao estresse crônico devido à maior exposição a fatores de risco (IRIGARAY, 2009).

Para finalizar a análise sociodemográfica, foi verificada uma alta prevalência da prática de exercícios. Há uma gama de evidências que associam a atividade física a melhorias significativas na habilidade funcional e na condição de saúde, prevenindo certas doenças ou diminuindo sua gravidade, porém é importante salientar que muitos desses benefícios requerem participação regular e contínua (MENDES; MORAES; GOMES, 2014). A sociedade e a população idosa já veem a atividade física como forma importante de prevenção e reabilitação da saúde.

Apesar de os sujeitos com CCL continuarem independentes na vida diária, eles encontram inúmeras dificuldades decorrentes das mudanças cognitivas, vivenciando grande estresse e isso pode acarretar mais prejuízo social, psicológico e na rotina (BANNINGH et al., 2008). Sabe-se também que há uma alta incidência de distúrbios do humor, como disforia, ansiedade, inquietude e irritabilidade.

Pacientes com CCL relatam perdas cognitivas subjetivas que podem ser objetivamente acessadas por meio de testes neuropsicológicos (COOPER et al., 2013). Não há um padrão-ouro que especifique qual bateria neuropsicológica deve ser usada, mas é importante que os principais domínios cognitivos sejam investigados; tipicamente, linguagem, memória e habilidades visuoespaciais (PETERSEN et al., 2014).

Existem poucos trabalhos que avaliem o desempenho no Addenbrooke de idosos com CCL; além disso, é comum que estes estudos associem o CCL a outras patologias, como a Doença de Parkinson, ou que se referiram a versões orientais do instrumento.

Sabe-se que os testes cognitivos sofrem influência de variáveis sociodemográficas, o que dificulta a interpretação dos resultados em populações heterogêneas como a brasileira. Assim, a adição de escalas funcionais pode resultar em um instrumento de alta sensibilidade e especificidade no rastreamento de prejuízos cognitivos, mesmo em uma população distinta do ponto de vista socioeconômico e cultural (PEREIRA et al., 2006). 
Atualmente, não há tratamento farmacológico ou não farmacológico recomendado para o CCL. Não foram observados, por exemplo, efeitos dos inibidores de colinesterase em testes cognitivos ou na progressão da demência durante três anos; por outro lado, exercícios físicos e treino cognitivo podem ter efeito neuroprotetor ou compensatório, melhorando o funcionamento cognitivo global e a memória (RUSS; MORLING, 2012). Como as monoterapias não têm demonstrado resultados satisfatórios, uma possibilidade é combinar diferentes tipos de tratamentos, avaliando se tal combinação é mais benéfica terapeuticamente do que uma intervenção isolada.

Nesse sentido, uma proposta de intervenção promissora é descrita por Bredesen (2014) e Bredesen et al. (2016). Consiste em um programa terapêutico personalizado que promove enriquecimento metabólico em quadros de neurodegeneração investigou pacientes com Doença de Alzheimer, CCL e Comprometimento Cognitivo Subjetivo (CCS). Os pesquisadores encontraram aumento no volume hipocampal e melhora nos testes neuropsicológicos. Tanto os pacientes quanto seus cônjuges e cuidadores identificaram melhoras significativas. Pacientes que haviam parado de trabalhar em razão das dificuldades cognitivas voltaram à ativa. Os pesquisadores apontaram que pacientes em estágios iniciais do Alzheimer ou com os seus precursores (CCL e CCS) podem se beneficiar substancialmente de terapias combinadas e personalizadas.

No tocante ao estado psicológico, sintomas de ansiedade e depressão são comuns em pacientes com CCL (ORGETA et al., 2007). Na presente amostra, as idosas com CCL apresentaram mais sintomas de ansiedade que as saudáveis. As participantes relataram preocupações diversas, especialmente em relação a problemas familiares e de saúde. Muitas também questionaram sobre a Doença de Alzheimer e tiveram dúvidas se as dificuldades cognitivas apresentadas no ACE-R têm alguma relação com a doença.

Os sintomas de ansiedade são um preditor da conversão do CCL amnéstico $^{7}$ para a Doença de Alzheimer, além de estarem relacionados com a atrofia do córtex entorrinal, uma das primeiras áreas cerebrais atingidas pelo Alzheimer. Esse achado corrobora com a hipótese de que a ansiedade não seja um fenômeno prodrômico não cognitivo, mas capaz de acelerar o declínio que conduz ao Alzheimer por meio de seus efeitos diretos e indiretos no córtex entorrinal (MAH et al., 2015).

A pontuação máxima da Escala de Desenvolvimento Pessoal é de 150 pontos e considera-se que quanto menor a pontuação do indivíduo, pior é a

7 É o subtipo mais comum de CCL e o que apresenta maior taxa de conversão para a Doença de Alzheimer (PELLEGRINO et al., 2013). 
condição psicológica (NERI, 2001). Os sujeitos com CCL mostraram pontuação inferior na EDEP, uma escala que não foi aplicada em estudos anteriores em pacientes com CCL. A literatura aponta que mesmo um declínio cognitivo leve está diretamente relacionado a queixas subjetivas de problemas psicológicos, os quais comumente estão associados à preocupação com a memória, sintomas de ansiedade e de depressão (GATES et al., 2011).

Por fim, o grupo com CCL obteve desempenho inferior aos controles nos instrumentos WHOQOL-bref e WHOQOL-old. Um estudo com quase 1.000 sujeitos (CONRAD et al., 2014) concluiu que pacientes com CCL apresentam piora significativa da qualidade de vida em relação a sujeitos normais. Pacientes com CCL têm sido alvo de estudos que propõem tratamentos precoces e preventivos. Instrumentos que avaliam bem-estar e qualidade de vida podem ser um indicador útil para a eficácia de tratamentos futuros (GATES et al., 2011), contexto no qual se insere a presente pesquisa.

Considerando o exposto, observamos que é frequente a queda da qualidade de vida e a piora no estado psicológico dos pacientes com CCL. Apesar das intervenções psicológicas serem vistas como potencialmente benéficas e fundamentais para que esses idosos enfrentem as dificuldades e desafios cotidianos, eles têm oportunidades muito limitadas de acessarem serviços psicoterapêuticos focados em melhorar seu bem-estar (ORGETA et al., 2007). Ademais, são necessários ensaios controlados, randomizados e de qualidade que incluam intervenções psicológicas em grupo como estratégia de intervenção para estes pacientes (COOPER et al., 2013).

É importante destacar que não foram encontrados na literatura estudos com idosos com CCL que incluíssem a bateria de instrumentos utilizada nessa pesquisa. Como ainda não há padrão-ouro para a avaliação neuropsicológica deste declínio cognitivo, a metodologia proposta neste trabalho pode ser um caminho.

Mais do que prejuízo cognitivo, esses idosos têm que conviver com piora do bem-estar psicológico, das relações sociais e da qualidade de vida. Ao demonstrar, de forma conjunta, como o CCL atingiu esses aspectos nos sujeitos estudados, a presente pesquisa contribui para a reflexão de possíveis estratégias de intervenção que busquem melhorar a condição geral desses pacientes ou mesmo reabilitar as áreas prejudicadas pela patologia. 
A principal contribuição desta pesquisa foi avaliar dados sobre a cognição, o estado psicológico e a qualidade de vida de idosos com Comprometimento Cognitivo Leve residente no Sul do Brasil. Não foi encontrada na literatura investigação desses idosos com os instrumentos utilizados neste estudo. Desta forma, a bateria de testes escolhida objetivou explicitar os danos diversos causados pelo CCL, com piora significativa nos cinco domínios cognitivos investigados, nos sintomas de ansiedade, no bem-estar psicológico e na qualidade de vida em relação a controles saudáveis. Em pesquisas posteriores, será possível utilizar esses instrumentos para mensurar a eficácia de diferentes modalidades terapêuticas em casos de declínio cognitivo.

Considerando-se que a avaliação neuropsicológica é uma ferramenta essencial para mensurar prejuízo e declínio das funções cognitivas e que não há padrão-ouro para o CCL, a metodologia proposta neste trabalho pode ser um caminho.

Em geral, as pesquisas da área avaliam idosos com histórico urbano e com escolaridade e renda mais altas. Esta amostra, entretanto, caracteriza-se por escolaridade e renda familiar predominantemente baixas, além de forte tradição rural, trazendo contribuições importantes se for considerada a heterogeneidade característica da população brasileira. São necessários mais estudos que investiguem as relações entre aspectos psicológicos, cognitivos e de qualidade de vida em sujeitos com CCL, incluindo-se o público masculino e aqueles que não participam de programas para idosos.

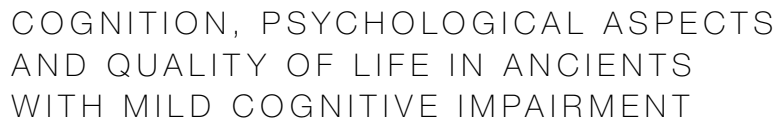

COGNITION, PSYCHOLOGICAL ASPECTS

AND QUALITY OF LIFE IN ANCIENTS WITH MILD COGNITIVE IMPAIRMENT

abstract

Mild Cognitive Impairment ( $\mathrm{MCl}$ ) is an intermediate state between normal cognition and dementia in which functional abilities are preserved. Objectives: To characterize a sample of CCL elderly living in southern Brazil in relation to the performance of instruments that evaluate cognition, psychological aspects and quality of life. Method: 96 elderly women aged 60 to 84 years were evaluated with the following instruments: Addenbrooke-Revised Cognitive Examination, Katz Index, Lawton \& Brody Scale, Geriatric Depression Scale, Beck Anxiety Inventory, Personal Development Scale, 
WHOQOL-bref and WHOQOL-old. The diagnosis of $\mathrm{MCI}$ was made from a semi-structured interview, cognitive and functional evaluation. Cognitive performance was adjusted according to the subjects' age and schooling. Results: The $\mathrm{MCl}$ group presented deficits in the five cognitive domains evaluated, anxiety symptoms, worse psychological well-being and quality of life. Conclusions: The combination of the instruments used in this study was not adopted and explicitly adopted for the various damages caused by $\mathrm{MCl}$. In general, as a research area evaluates elderly with urban history, and higher education and income. This sample, however, is characterized by predominantly low schooling and family income, as well as a strong rural tradition, bringing important contributions that consider a characteristic heterogeneity of the Brazilian population. Considering that a neuropsychological evaluation is an essential tool to measure the impairment and decline of cognitive functions and that it is not the gold standard for $\mathrm{MCl}$, the methodology proposed in this work may be a path.

keywords

Mild Cognitive Impairment. Anxiety. Depression. Quality of Life. Aged.

referências

ALMEIDA, Osvaldo P.; ALMEIDA, Shirley A. Confiabilidade da versão brasileira da Escala de Depressão em Geriatria (GDS) versão reduzida. Arquivos de Neuro-Psiquiatria, São Paulo, v. 57, n. 2B, p. 421-426, jun. 1999.

BANNINGH, Liesbeth Joosten-Weyn et al. Mild cognitive impairment: coping with an uncertain label. International Journal of Geriatric Psychiatry, Chichester, v. 23, n. 2 , p. 148-154, Feb. 2008.

BARROS, Marilisa Berti de Azevedo et al. Social inequalities in health among the elderly. Cadernos de Saúde Pública, Rio de Janeiro, v. 27, supl. 2, p. s198-s208, 2011.

BATISTA, Nancy Nay Leite de Araújo Loiola; VIEIRA, Débora Josefina Neiva; SILVA, Gislene Mariana Pereira da. Caracterização de idosos participantes de atividade física em um centro de convivência de Teresina-Pl. Enfermagem em Foco, Salvador, v. 3, n. 1, p. 7-11, 2012.

BREDESEN, Dale E. Reversal of cognitive decline: A novel therapeutic program. Aging, Albany, NY, v. 6, n. 9, p. 707-717, Sept. 2014

BREDESEN, Dale E. et al. Reversal of cognitive decline in Alzheimer's disease. Aging, Albany, NY, v. 8, n. 6, p. 1250-1258, June 2016.

CARLOMANHO, Aline Murari Ferraz; SOARES, Edvaldo. Declínio cognitivo e depressão em idosos institucionalizados e não institucionalizados: possibilidades de correlação. Revista de Iniciação Científica da FFC, Marília, v. 13, n. 3, p. 1-23, 2013. 
CARVALHO, Viviane Amaral; CARAMELLI, Paulo. Normative Data for Healthy MiddleAged and Elderly Performance on the Addenbrooke Cognitive Examination-Revised. Cognitive and Behavioral Neurology, Hagerstown, MD, v. 25, n. 2, p. 72-76, June 2012.

Brazilian adaptation of the Addenbrooke's cognitive examination-revised (ACE-R). Dementia \& Neuropsychologia, São Paulo, v. 1, n. 2, p. 212-216, Apr./May/ June 2007.

CONRAD, Ines et al. Quality of Life of Individuals with Mild Cognitive Impairment. Psychiatrische Praxis, Stuttgart, v. 42, n. 3, p. 152-157, Feb. 2014.

COOPER, Claudia et al. Treatment for Mild Cognitive Impairment: Systematic Review. British Journal of Psychiatry, London, v. 203, n. 3, p. 255-264, Sept. 2013.

CUNHA, Jurema Alcides et al. Psicodiagnóstico V. Porto Alegre: Artmed, 2000.

DENNIS, Michael; KADRI, Adil; COFFEY, John. Depression in older people in the general hospital: a systematic review of screening instruments. Age and Ageing, Oxford, v. 41, n. 2, p. 148-154, Mar. 2012

FLECK, Marcelo P. A. et al. Aplicação da versão em português do instrumento abreviado de avaliação da qualidade de vida "WHOQOL-bref". Revista de Saúde Pública, São Paulo, v. 34, n. 2, p. 178-183, abr. 2000.

GATES, Nicola J. et al. Cognitive and memory training in adults at risk of dementia: A Systematic Review. BMC Geriatrics, London, v. 11, p. 55, Sept. 2011.

GOLD, David A. An examination of instrumental activities of daily living assessment in older adults and mild cognitive impairment. Journal of Clinical and Experimental Neuropsychology, Lisse, v. 34, n. 1, p. 11-34, July 2012.

INSTITUTO BRASILEIRO DE GEOGRAFIA E ESTATÍSTICA (IBGE). Projeção da população do Brasil e das unidades da Federação. Rio de Janeiro, 2013. Disponível em: <http:// www.ibge.gov.br/apps/populacao/projecaos. Acesso em: 10 maio 2015.

IRIGARAY, Tatiana Quarti. Efeitos de um treino de atenção, memória e funções executivas na cognição, na qualidade de vida e no bem-estar psicológico de idosos saudáveis. 2009. 129 f. Tese (Doutorado em Gerontologia Biomédica) - Instituto de Geriatria e Gerontologia, Pontifícia Universidade Católica do Rio Grande do Sul, Porto Alegre, 2009.

KRUG, Rodrigo de Rosso; LOPES, Marize Amorim; MAZO, Giovana Zarpellon. Características sociodemográficas e condições de saúde de idosas longevas inativas fisicamente participantes de grupos de convivência para idosos. Revista Kinesis, Santa Maria, v. 32, n. 1, p. 6-22, jan./jun. 2014.

LINO, Valéria Teresa Saraiva et al. Adaptação transcultural da Escala de Independência em Atividades da Vida Diária (Escala de Katz). Cadernos de Saúde Pública, Rio de Janeiro, v. 24, n. 1, p. 103-112, jan. 2008.

$\mathrm{MAH}$, Linda et al. Anxiety symptoms in amnestic mild cognitive impairment are associated with medial temporal atrophy and predict conversion to Alzheimer disease. American Journal of Geriatric Psychiatry, London, v. 23, n. 5, p. 466-476, May 2015

MENDES, Gisele Soares; MORAES, Clayton Franco; GOMES, Lucy. Prevalência de hipertensão arterial sistêmica em idosos no Brasil entre 2006 e 2010. Revista Brasileira de Medicina de Familia e Comunidade, Rio de Janeiro, v. 9, n. 32, p. 273-278, jul./set. 2014.

NERI, Anita Liberalesso (Org.). Palavras-chave em Gerontologia. Campinas: Alínea, 2001.

ORGETA, Vasiliki et al. Psychological treatments for depression and anxiety in dementia and mild cognitive impairment: systematic review and meta-analysis. British Journal of Psychiatry, London, v. 207, n. 5, p. 293-298, Oct. 2007.

PELLEGRINO, Laurel D. et al. Depression in Cognitive Impairment. Current Psychiatry Reports, Philadelphia, PA, v. 15, n. 9, p. 384, Sept. 2013. 
PEREIRA, Renata Junqueira et al. Contribuição dos domínios físico, social, psicológico e ambiental para a qualidade de vida global de idosos. Revista de Psiquiatria do Rio Grande do Sul, Porto Alegre, v. 28, n. 1, p. 27-38, jan./abr. 2006.

PETERSEN, Ronald C. et al. Mild cognitive impairment: a concept in evolution. Journal of Internal Medicine, Oxford, v. 275, n. 3, p. 214-228, Mar. 2014.

POWER, Mick; QUINN, Kathryn; SCHMIDT, Silke. Development of the WHOQOL-Old Module. Quality of Life Research, Amsterdam, v. 14, n. 10, p. 2197-2214, Dec. 2005.

REIS, Luciana Araújo dos; REIS, Luana Araújo dos; TORRES, Gilson de Vasconcelos. Impacto das variáveis sociodemográficas e de saúde na capacidade funcional de idosos de baixa renda. Ciência, Cuidado e Saúde, Maringá, v. 14, n. 1, p. 847-854, jan./mar. 2015.

RUSS, Tom C.; MORLING, Joanne. Cholinesterase inhibitors for mild cognitive impairment. Cochrane Database of Systematic Reviews, Chichester, v. 9, n. 10, p. CD009132, Sept. 2012.

SANTOS, Roberto Lopes dos; VIRTUOSO JÚNIOR, Jair Sindra. Confiabilidade da versão brasileira da escala de atividades instrumentais da vida diária. Revista Brasileira em Promoção da Saúde, Fortaleza, v. 21, n. 4, p. 290-296, jan. 2008.

SMAGULA, Stephen F. et al. Risk factors for sleep disturbances in older adults: evidence from prospective studies. Sleep Medicine Reviews, London, v. 25, p. 21-30, Feb. 2016

STOKIN, Gorazd B. et al. Mild Neurocognitive Disorder: An Old Wine in a New Bottle. Harvard Review of Psychiatry, Philadelphia, PA, v. 23, n. 5, p. 368-376, Sept./Oct. 2015

WORLD HEALTH ORGANIZATION (WHO). The Heidelberg guidelines for promoting physical activity among older persons. Geneva, 1996. p. 1-12. Avaliable from: <http:// apps.who.int/iris/bitstream/10665/108545/1/E75205.pdf>. Cited: 20 set. 2015.

ZAVATINI, Márcia Adriana; OBRELI NETO, Paulo Roque; CUMAN Roberto Kenji Nakamura. Estratégia saúde da família no tratamento de doenças crônico-degenerativas: avanços e desafios. Revista Gaúcha de Enfermagem, Porto Alegre, v. 31, n. 4 , p. 647-654, dez. 2010. 
